\title{
Jejunum and Ileum Neuroendocrine Tumor pT4 TNM Finding v8
}

National Cancer Institute

\section{Source}

National Cancer Institute. Lejunum and Ileum Neuroendocrine Tumor pT 4 TNM Finding v8. NCI Thesaurus. Code C135113.

Jejunum and ileum neuroendocrine tumor invading visceral peritoneum (serosa) or other organs or adjacent structures. (from AJCC 8th Ed.) 